\title{
Vaccum-Assisted Closure Therapy in Split-Thickness Skin Graft on the Wound on the Contours of the Body
}

\author{
Seung Bum Pyo ${ }^{1}$, Kun Yong Sung ${ }^{2}$, Hong Sil Joo ${ }^{1}$, Jin Kyung Song ${ }^{1}$, Seong Yoon Lim ${ }^{1}$ \\ ${ }^{1}$ Department of Plastic and Reconstructive Surgery, Hanil General Hospital, Seoul; ${ }^{2}$ Department of Plastic and Reconstructive Surgery, College of Medicine, \\ Kangwon National University, Kangwon National University Hospital, Chuncheon, Korea
}

\begin{abstract}
Background: For successful skin grafting, contact between the graft and the wound bed is important. To promote close contact between the graft and the wound, a splint or bolster dressing was applied to the graft site. However, the splint could not be fit into wound on the contours of the body. Patients complained about the use of splint due to their inconvenience. Therefore, we present the method of a vacuum-assisted closure (VAC) therapy, a replacement for the use of a splint on the graft site, that can improve graft survival rate on wounds on the contours of the body.

Methods: Through a chart review, we studied medical records from January 2014 to July 2017. Wounds on the contours of the body of 106 patients were healed with VAC therapy following skin graft. The data was collected for characteristics such as age, sex, size of wounds, and underlying disease, complications.

Results: Among 106 patients, there was 1 patient for face, 2 for neck, 6 for anterior chest, 1 for shoulder, 2 for axilla, 1 for back, 8 for arm, 3 for hand, 1 for sacrum, 3 for buttock, 2 for trochanter, 9 for thigh, 27 for lower leg, 43 for foot. There were 4 patients for active bleeding, 5 for minor hematoma, 2 for partial graft loss.

Conclusion: VAC therapy reduced graft loss and improved graft success rate due its close contact ability. The patient was satisfied with the ease of dressing and handiness. In addition, patients and medical staff have satisfactory results due to increased mobility during hospitalization.
\end{abstract}

Keywords: Negative-pressure wound therapy, Skin graft, Splint

\section{Introduction}

A skin graft is a process in which the skin tissue is taken from the donor site and placed over the graft site. The engraftment of a skin graft is influenced by many diverse factors and the revascularization stage, which occurs within 24-48 hours after grafting, is known as the key point for the long-term success of grafting. Therefore, it is important that the graft is fixed without any movements at this stage. To date, the existing method of bolster dressing in parallel with application of a splint is used to limit the movements of the grafted part and for effective fixation. However, if a splint has been applied, it can produce pain or discomfort in the patient, and if grafting is performed on the curved body parts like the neck, shoulder, breast, hips, and axilla, it is difficult to shape the split to fit the body curve. Therefore, this research attempted to achieve an effective result of negative pressure wound therapy without applying a splint after skin grafting on the wounds on the contours of the body and to report this new method.

\section{Methods}

Materials

Through investigation of the recording, the testing group included 106 patients, who

\section{Original Article}

Received: August 23, 2017

Revised: September 19, 2017

Accepted: September 20, 2017

\section{Corresponding author:}

Hong Sil Joo, M.D., Ph.D.

Department of Plastic and Reconstructive Surgery, Hanil General Hospital, 308 Uicheonro, Dobong-gu, Seoul 01450, Korea

Tel: +82-2-901-3109

Fax: +82-2-901-3104

E-mail: sil9113@gmail.com

No potential conflict of interest relevant to this article was reported.

This is an Open Access article distributed under the terms of the Creative Commons Attribution Non-Commercial License (http://creativecommons.org/licenses/by-nc/4.0/) which permits unrestricted non-commercial use, distribution, and reproduction in any medium, provided the original work is properly cited.

(c) 2017 Korean Wound Management Society 
were hospitalized at Department of Plastic Surgery of Hanil General Hospital from January 2014 to July 2017 and underwent negative pressure wound therapy parallel to the grafting part. This paper investigates gender, age, part of surgery, cause of injury, underlying disease, size of the surgical area, complications after surgery etc.

\section{Surgical methods}

Operations were performed in the same manner in all of the patients. First of all, the grafting part was collected by using a dermatome (Zimmer ${ }^{\circledR}$, Air dermatome, Zimmer Surgical Inc., Warsaw, IN, USA) from the thigh at a 10/1,000 inch thickness. After placing the meshed graft on the receiving part, in which the margin tissue had been eliminated, it was fixed by using a stitching fiber and a medical stapler. For better release of the hematoma or exudation, Mepitel ${ }^{\circledR}$ (Molnlycke Health Care, Box 13080, SE-402 52 Goteborg, Sweden) was applied on top of the graft and then, the antibiotic ointment was applied. Lastly, for negative pressure wound therapy, the V.A.C. ${ }^{\mathrm{TM}}$ system ( $\mathrm{KCl}$ inc., San Antonio, USA) and Curavac system (CGBio, Sungnam, Korea) were used in operation. Immediately after the operation, the pressure of the vacuum pump was set to $75 \mathrm{mmHg}$ in a continuous mode for decreasing bleeding when there was active bleeding. When there was no active bleeding and exudation and it stabilized, the pressure was set to $100 \mathrm{mmHg}$ for promoting absorption exudate and bleeding. After that, negative pressure wound therapy was maintained for 3 days, if there was no complications such as pain, and bleeding.

\section{Results}

All of the 106 patients underwent negative pressure wound therapy after skin grafting, and among them, there were 66 male patients and 40 female patients. With respect to the injured areas, the face was involved in 1 case, the neck was involved in 2 cases, the anterior chest including breasts was involved in 6 cases, the shoulder was involved in 1 case, axillae were involved in 2 cases, the back was involved in 1 case, the arm was involved in 8 cases, the hand was involved in 3 cases, the sacrum was involved in 1 case, buttocks were involved is 3 cases, trochanteric area was involved is 2 cases, the thigh was involved in 9 cases, the lower leg was involved in 27 cases, the foot except for the toes was involved in 30 cases, and the foot including the toes was involved in 13 cases. The average size of the operated wound area was 68.4 $\mathrm{cm}^{2}$, and among these areas, the maximum size was 625 $\mathrm{cm}^{2}$. With respect to the cause of injury, scalding burns occurred in 60 patients, contact burns occurred in 20 patients, flame burns occurred in 8 patients, electrical burns occurred

Table 1. Clinical characteristics

\begin{tabular}{|c|c|}
\hline Variable & Total $(n=106)$ \\
\hline \multicolumn{2}{|l|}{ Gender } \\
\hline Man & $66(62.3 \%)$ \\
\hline Woman & $40(37.7 \%)$ \\
\hline Patient Age (year) & $49.5 \pm 21.6$ \\
\hline \multicolumn{2}{|l|}{ Site of wound } \\
\hline Face & $1(0.9 \%)$ \\
\hline Neck & $2(1.8 \%)$ \\
\hline Anterior chest & $6(5.5 \%)$ \\
\hline Shoulder & $1(0.9 \%)$ \\
\hline Axilla & $2(1.8 \%)$ \\
\hline Back & $1(0.9 \%)$ \\
\hline Arm & $8(7.3 \%)$ \\
\hline Hand & $3(2.8 \%)$ \\
\hline Sacrum & $1(0.9 \%)$ \\
\hline Buttock & $3(2.8 \%)$ \\
\hline Trochanter & $2(1.8 \%)$ \\
\hline Thigh & $9(8.3 \%)$ \\
\hline Lower leg & $27(24.8 \%)$ \\
\hline Foot & $30(27.5 \%)$ \\
\hline Foot (including toes) & $13(11.9 \%)$ \\
\hline Size of wound $\left(\mathrm{cm}^{2}\right)$ & $68.4 \pm 103.6$ \\
\hline Maximum size & 625 \\
\hline Minimum size & 1 \\
\hline \multicolumn{2}{|c|}{ Mode of injury (patients number) } \\
\hline Scalding burn & $60(56.6 \%)$ \\
\hline Contact burn & $20(18.9 \%)$ \\
\hline Flame burn & $8(7.5 \%)$ \\
\hline Electrical burn & $5(4.7 \%)$ \\
\hline Chemical burn & $1(0.9 \%)$ \\
\hline Pressure sore & $4(3.8 \%)$ \\
\hline Skin defect & $6(5.7 \%)$ \\
\hline Burn scar contracture & $2(1.9 \%)$ \\
\hline \multicolumn{2}{|c|}{ Comorbidities (patients number) } \\
\hline Hypertension & $23(21.7 \%)$ \\
\hline Diabetes mellitus & $21(19.8 \%)$ \\
\hline \multicolumn{2}{|c|}{ Complications (patients number) } \\
\hline Active bleeding & $4(3.8 \%)$ \\
\hline Minor hematoma & $5(4.7 \%)$ \\
\hline Partial graft loss & $2(1.9 \%)$ \\
\hline
\end{tabular}


in 5 patients, a chemical burn occurred in 1 patient, a pressure sore occurred in 4 patients, skin defect occurred in 6 patients, and burn scar contractures occurred in 2 patients. With respect to the underlying disease, which the patients had before the accident, hypertension was noted in 23 patients and diabetes was noted in 21 patients. There was 4 cases of active bleeding, 5 cases of minor hematoma, and 2 cases of partial loss of the graft part as complications after grafting (Table 1).

\section{Case 1}

A 29-year-old male patient, who had no underlying disease, injured his neck area by coming in contact with heater fire.
Afterwards, split-thickness skin graft was performed over this skin area and negative pressure wound therapy was simultaneously performed. At three days after surgery, the vacuumassisted closure was removed and it was confirmed that the skin graft was well fixed without any distinct hematoma and exudate (Fig. 1). He recovered with the disinfection and antibiotic treatment without any loss of graft.

\section{Case 2}

A 59-year-old female patient, who had no underlying disease, injured her anterior chest including the left breast with boiling water. A split-thickness skin graft was performed in parallel with negative pressure wound therapy. At three days after
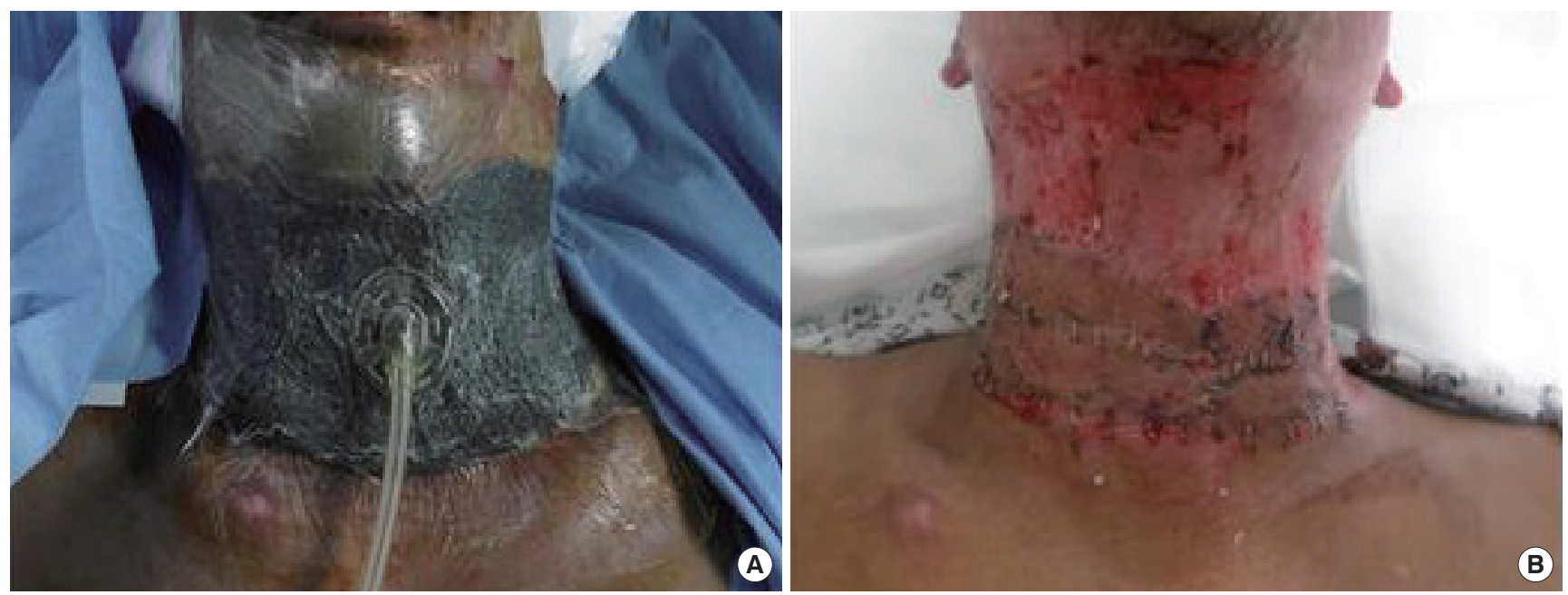

Fig. 1. (A) The flame burn wound on neck covered with skin graft using vacuum-assisted closure therapy. (B) Postoperative photograph of neck without complications such as hematoma or exudate.
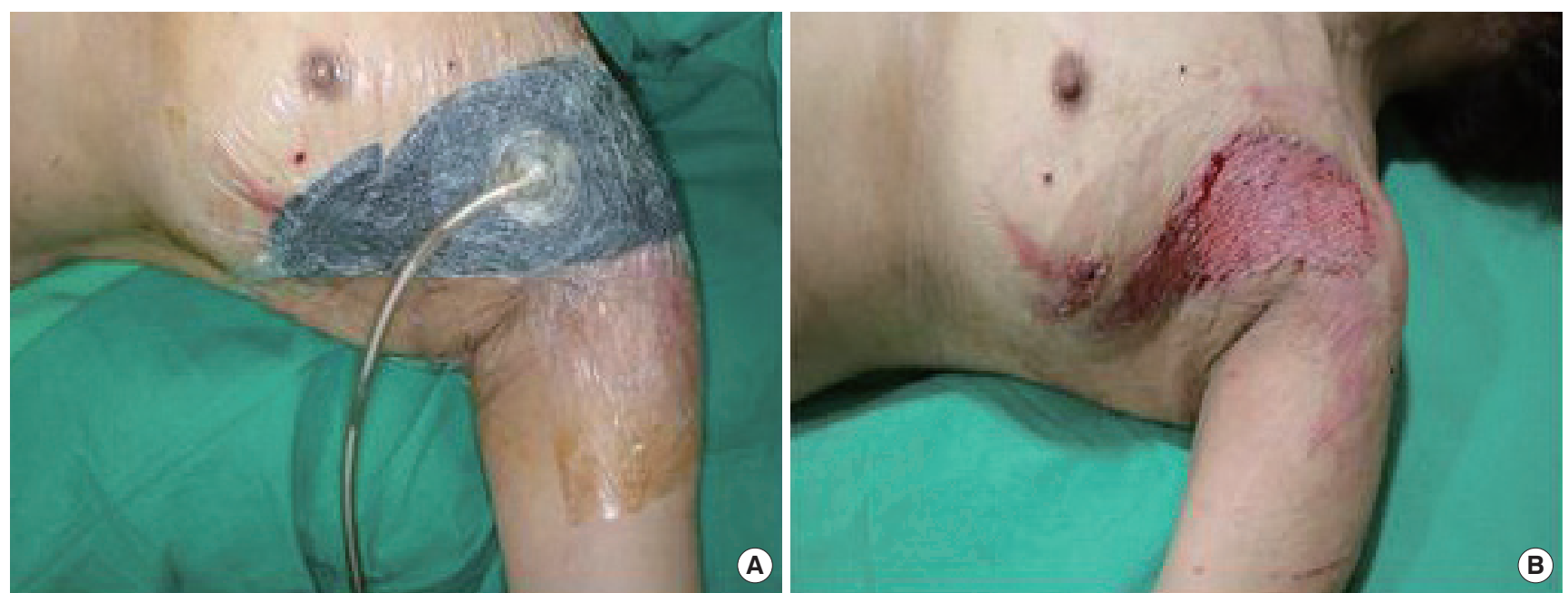

Fig. 2. (A) The scalding burn wound on left anterior chest including breast covered using skin graft and vacuum-assisted closure therapy. (B) Postoperative photograph of left anterior chest including breast. 
surgery, the vacuum-assisted closure was removed, and the skin graft was well-fixed without any hematoma or exudation (Fig. 2).

\section{Disscusion}

Skin grafting occurs over a maximum period of 1 year and it includes the processes of serum imbibition, revascularization, and maturation. Among these processes, revascularization is one of the most critical factors for the long-term success of grafting [1]. If a hematoma or exudation under the graft occurs or there is any indication like an accompanying infection because of improper fixation of the skin graft, it could cause graft loss [3]. Therefore, it is important to limit the movement and to fix the skin graft within this time period. In order to achieve this, a bolster dressing and fixation of a splint have been mainly used until now. Bolster dressing, which fixes directly on the skin graft, has an effect, which prevents loss of the graft by a hematoma or exudation. However, there are some shortcomings as the narrow surface and wounds on the contours of the body like fingers or toes are difficult to disinfect and the hospitalization period could be prolonged [4]. Fixing of the splint can secure the skin graft by limitation of joint movements in case of a skin graft placed around the joint area. However, it is difficult to apply a splint precisely to the wound on the contours of the body like the neck, shoulder, breast, hip, and axilla. A imprecisely placed splint can cause pain in patients and there is a risk of development of a pressure sore. Also, the splint can limit light movements of patients in daily life [2]. Moreover, if the fixation of a splint is not properly performed, there is more risk of graft loss because of the improper fixation of a skin graft. Therefore, in this research, we attempted negative pressure wound therapy in parallel with skin grafting, which can lead to proper engraftment of the skin graft, to overcome the existing disadvantage of the dressing treatment.

Generally, application of negative pressure wound therapy to the wound reduces the number of bacterial colonies and improves the cell-mediated immune reaction. It also helps in recovery of the wound by decreasing the vascular permeability, assisting in vascularization, decreasing exudation from the wound, and stimulating the formation of granulation tissues [5].

When negative pressure wound therapy is performed on the graft, infection, which has an influence on failure of skin grafting, can be controlled because bacterial multiplication can be restrained. Therefore, it can reduce the loss of the skin graft [6]. In this research, there was no case of loss of the graft due to infection. Moreover, grafting can be performed smoothly by increasing the blood flow in the grafting area by performing negative pressure wound therapy. Compared to the existing dressing treatment, it can decrease the loss of graft due to better fixation of the graft because exudation and hematoma are easily absorbed [4]. And it is more comfortable than bolster dressing because of its easiness to apply on wound. As a result, the length of treatment on operating room can be reduced [7].

For negative pressure wound therapy, polyurethane foam is used. By elimination of air in the polyurethane foam, it will be condensed and will induce an effect to compress the graft. Due to this reason, we achieve the effect of decreasing the dead space on the curvature of the body, which exists under the polyurethane foam. Also, it can be applied on the large burn area that is difficult to use conventional bolster dressing [2]. Therefore, when negative pressure wound therapy is performed, we achieve the splinting effect without applying the splint [8].

When negative pressure wound therapy is performed in parallel with skin grafting on the wounds on the contours of the body, the patient satisfaction rate increases because of increased patient mobility and it can achieve an effect, which increases the convenience for the medical team because of better convenience in comparison with the existing dressing treatment. In addition, postoperative-management becomes more convenient than that with the former procedure and the hospitalization period is reduced [6,9].

If the grafted skin is dressed by the former bolster dressing after skin grafting, the failure rate is generally reported to range from 15\% to 30\% [10]. In this report, the rate of partial loss of the graft was about $2 \%$ with negative pressure wound therapy after fixation of the skin graft, and all of the patients were discharged from the hospital without any reoperation.

Recently there was a case, in which the hospitalization period was shortened by increasing the graft success rate with negative pressure wound therapy after split-thickness skin grafting in comparison with the former treatment [10]. Former researches have reported the effectiveness of performing negative pressure wound therapy in parallel with skin grafting. But this research attempted to report the utility of performing negative pressure wound therapy in parallel with skin grafting on the wounds on the contours of the body, which are difficult to care for by applying a splint and the existing 
dressing treatment. Negative pressure wound therapy can be performed with donor site dressing by local flap coverage and other variable flap coverage methods [11]. Therefore, in the future, the effect when negative pressure wound therapy is performed in parallel with other operations except for a skin graft should be discussed.

\section{Conclusion}

When negative pressure wound therapy is performed in parallel with skin grafting on the curved body parts, the engraftment of the skin graft is better than before and the loss of the graft is further decreased than the case in which the existing dressing treatment is used. Moreover, patients could be satisfied due to better mobility after negative pressure wound therapy and the effectiveness of the medical team is increased because the dressing is easier than with the former method.

\section{References}

1. Scherer S, Pietramaggiori G, Orgill DP. Skin graft. In: PC Neligan, Plastic surgery, 3rd ed, USA: Elservier, 2012;323-4.

2. Hoeller M, Schintler MV, Pfurtscheller K, Kamolz LP, Tripolt N, Trop M. A retrospective analysis of securing autologous splitthickness skin grafts with negative pressure wound therapy in paediatric burn patients. Burns 2014;40:1116-20.

3. Mohsin M, Zargar HR, Wani AH, et al. Role of customised negative-pressure wound therapy in the integration of splitthickness skin grafts: a randomised control study. Indian J
Plast Surg 2017;50:43-9.

4. Azzopardi EA, Boyce DE, Dickson WA, et al. Application of topical negative pressure (vacuum-assisted closure) to splitthickness skin grafts: a structured evidence-based review. Ann Plast Surg 2013;70: 23-9.

5. Borgquist O, Ingemansson R, Malmsjö M. The influence of low and high pressure levels during negative-pressure wound therapy on wound contraction and fluid evacuation. Plast Reconstr Surg 2011;127:551-9.

6. Henderson NJ, Fancourt M, Gilkison W, Kyle S, Mosquera D. Skin grafts: a rural general surgical perspective. ANZ J Surg 2009;79:362-6.

7. Kim EK, Hong JP. Efficacy of negative pressure therapy to enhance take of 1-stage allodermis and a split-thickness graft. Ann Plast Surg 2007;58:536-40.

8. Peter Suh HS, Hong JP. Effects of Incisional Negative-Pressure Wound Therapy on Primary Closed Defects after Superficial Circumflex lliac Artery Perforator Flap Harvest: Randomized Controlled Study. Plast Reconstr Surg 2016;138: 1333-40.

9. Jung HG, Kee SC, Kim BH, et al. Application of Vacuum-assisted Closure in Difficult Wounds. J Korean Orthop Assoc 2012;47:41-7.

10. Lee DH, Kim YJ. Negative Pressure Wound Therapy Applied to a Meshed Split-Thickness Skin Graft. Arch Reconstr Microsurg 2016;25:29-36.

11. Nguyen TQ, Franczyk M, Lee JC, Greives MR, O'Connor A, Gottlieb LJ. Prospective randomized controlled trial comparing two methods of securing skin grafts using negative pressure wound therapy: vacuum-assisted closure and gauze suction. J Burn Care Res 2015;36:324-8. 